\title{
A New Diffusion Scheme in Vortex Methods for Three-Dimensional Incompressible Flows
}

\author{
S. Shankar and L.L. van Dommelen \\ Department of Mechanical Engineering \\ FAMU/FSU College of Engineering \\ PO Box 2175, Tallahassee, Florida, 32316, USA
}

\begin{abstract}
We propose and validate a new, accurate, robust, mesh-free method to represent diffusion processes in Lagrangian computations, called "vorticity redistribution". The basic idea of this method is to represent diffusion by modifying the strength of the vortices: circulation is exchanged between vortices in the immediate vicinity of one another in such a way that the correct diffusion is achieved. Our method resolves length scales up to the spacing of the vortices, and it has no inherent limitations on the order of accuracy. Moreover, conservation of circulation, linear and angular momentum are implicit in the formulation. For its validation, we compute the flow over an impulsively started cylinder at $R e=9500$ (based on diameter); we compare the vorticity field with another recent high resolution simulation. The method is extended to three-dimensions and tested on two linear problems.
\end{abstract}

\section{Introduction}

Vortex methods are numerical methods to solve the vorticity transport equation of incompressible flow. They take advantage of the fact that vorticity is a material quantity in inviscid flows. For two-dimensional flows, the vorticity distribution is approximated by a superposition of discrete vortices. These vortices move according to the local velocity field and diffuse by fluid viscosity. In three-dimensional flows, vortices also change strength due to vortex stretching or tilting. Excellent surveys of the vortex method and its applications are given in $[2,4,10,11,12,13]$ and references therein.

For an efficient, high-resolution Lagrangian computation, both convection and diffusion have their unique problems. Representation of the convective processes in a vortex computation can be done by moving the vortices with the local flow velocity. To do that, fast algorithms were developed by, among others, Greengard and Rokhlin [8] and Carrier et al. [5], and independently by van Dommelen and Rundensteiner [17]. Our computations use the latter of these three schemes. For all these schemes, the required amount of work is roughly proportional to $N$. More recent variations have been proposed by a number of authors, such as $[1,3]$.

Certainly for two-dimensional flows, fast summation procedures made the accurate numerical representation of convection possible for large numbers of vortices. Hence, handling the diffusion processes became the main bottleneck. The main difficulty is the highly irregular distribution of the vortices generated by the vortex motion. We have recently developed a new 'vorticity redistribution' method which addresses this problem effectively.

In the following sections we outline the the basic idea of the 'vorticity redistribution' method and summarize its unique features. For the mathematical aspects and detailed comparisons of 
this method with other schemes, please refer to $[14,16]$. We also present numerical results in two-dimensions for further validation and for two linear problems in three-dimensions.

\section{Vorticity redistribution method}

Our redistribution method simulates diffusion during each time step by spreading the vorticity of each vortex over the neighboring vortices. In this respect our method is similar to the deterministic particle methods such as those of Degond and Mas-Gallic [6] and Fishelov [7]. However, there is an essential difference. The particle methods fix the amounts of vorticity to transfer between vortices using a chosen function. Instead, we formulate a system of equations for the amounts to transfer. In the next subsection we explain our procedure, then we discuss the advantages that can be obtained by computing the amounts of vorticity to transfer, instead of assuming them.

\subsection{General procedure}

In this section we summarize the actual steps in the redistribution method. A more complete discussion is given in $[14,16]$. To simulate the diffusion during a time-step $\Delta t$, first we identify for each vortex the neighboring vortices within a distance $R \sqrt{\nu \Delta t}$, where we took $R=\sqrt{12}$ (see Fig. 1).

Next, we determine the vorticity fractions to be transferred toward the neighboring vortices. We will indicate these fractions by $f_{i j}^{n}$, where $i$ is the vortex being diffused, $j$ is the receiving vortex and $n$ is the time-level. From a Fourier transform argument, appropriate equations for the fractions $f_{i j}^{n}$ are found from the vortex positions as (see $\left.[14,16]\right)$ :

$$
\begin{aligned}
O(1): & \sum_{j} f_{i j}^{n}=1 \quad ; \\
O(\Delta t)^{1 / 2}: & \sum_{j} f_{i j}^{n}\left(x_{k i}-x_{k j}\right)=0 \quad, \quad(k=1,2,3) ; \\
O(\Delta t): & \sum_{j} f_{i j}^{n}\left(x_{k i}-x_{k j}\right)\left(x_{l i}-x_{l j}\right)=2 \sqrt{\nu \Delta t} \delta_{k l}, \\
& (k, l=1,2,3) .
\end{aligned}
$$

When the transferred fractions of vorticity satisfy these equations, diffusion is correctly represented. As shown, the scheme is first order accurate. More equations can be added to the ones above to obtain higher accuracy.

To ensure stability, we require that the fractions $f_{i j}^{n}$ are positive. Typically, we have more unknown fractions $f_{i j}^{n}$ than equations to satisfy, so that we need to find a positive solution to an underdetermined system of equations. This problem can be handled by linear programming methods $[14,16]$. The computational time involved is unimportant due to the relatively large computational effort required to find the velocity field. If there are not enough neighboring vortices within the prescribed distance, or there are no positive solutions to the above equations, new vortices of zero strength are added. These new vortices are placed in the largest 'hole' in the vorticity distribution near the considered vortex: we try a few possible locations and choose the location that is farthest from the existing vortices $[14,16]$.

Shankar and van Dommelen $[14,16,18]$ show analytically that the method converges for at least the linear Stokes equations. Numerical results $[14,15,16,19,20]$ indicate that it also converges for the Navier-Stokes equations. 


\subsection{Properties of the method}

- Mesh-free property:

The redistribution method does not require a regular point distribution or even an ordering of the vortices. The only requirement is to identify the neighboring vortices. Thus the method is much more flexible and simpler to implement than the deterministic particle methods, especially for complex geometries. Treatment of boundary conditions is also simplified.

The accuracy of other methods deteriorates as the particles become disordered due to convection. These methods use remeshing to maintain accuracy for longer times. This introduces additional errors, uncertainties and programming complexities. We do not face such difficulties, since our procedure automatically adds new vortices of zero strength where needed.

\section{- Resolution:}

The redistribution method allows the vorticity to be spread over only a finite number of neighbors, while for other methods the number of neighbors must be asymptotically infinite. Spreading the vorticity over a small area allows us to resolve sharper gradients. It also simplifies the treatment of boundaries.

- Choice of smoothing function:

To evaluate the continuous vorticity and velocity fields from the computed vortex strengths, we use a smoothing function. However, our actual computation is independent of this function. It can, therefore, be chosen solution-adaptively to best represent the computed data. In contrast, the particle method computations do depend on an a priori choice of smoothing function. Since less is known about the solution beforehand, an optimal or solution adaptive choice may not be simple using these methods.

- Conservation laws:

The redistribution method satisfies conservation laws exactly.

- Order of accuracy:

The redistribution method can, in principle, have any order of accuracy. However, so far only first order accurate solutions have been implemented.

- Positivity:

The redistribution method will preserve the sign of a uniformly positive or negative vorticity field.

- Computational efficiency:

The redistribution method only needs vortices where there is vorticity. New vortices are added automatically when the vorticity diffuses. This reduces the number of vortices required and hence the computational time.

Our implementation in its present form can most likely still be improved dramatically $[14,16]$. However, it is fully functional and already gives excellent results.

\section{Numerical results}

This section presents some actual results of vortex computations that demonstrate the accuracy and practical usefulness of the method. 


\subsection{Diffusing point vortices}

The flow due to a single diffusing point vortex has a simple exact solution. Although convection in that flow is trivial for the exact solution, it is not for the vortex computation, making it a fair test. Tests $[14,16]$ show that the redistribution method performs excellently.

As a second example, we consider the flow due to a counter-rotating vortex pair. For this flow, the convection effects are non-trivial even for the analytical solution. The initial flow consists of two counter-rotating point vortices spaced a unit distance apart. The vortices diffuse out in time, while the vortex system also drifts in the direction normal to the line connecting the vortex centers. The Reynolds number $(R e)$, will be defined as $\Gamma / 2 \pi \nu$ with $\Gamma$ the initial circulation of the vortices, and the flow is normalized to have a unit initial drift velocity. We will further use a scaled viscous time $\tau \equiv \nu t$. In our computation we used a time step $\Delta \tau=0.00025$, an average spacing between the vortices $\sqrt{6 \Delta \tau}$, and a maximum distance between neighbors of $\sqrt{12 \Delta \tau}$.

Figure 2 shows the distribution of the computational vortices at various times, for $R e=50$. Our computation starts with only two vortices, but our method adds computational vortices automatically when the vorticity field diffuses out. Notice that these vortices are unordered and irregularly spaced. The small circle in the bottom right frame shows the size of the neighborhood over which the vortices are diffused. The size of this circle is a measure of the resolution in our computation.

For the above flow no exact analytical solution is available. However, the numerical results can be compared to the asymptotic expansion derived in [19], which is valid for sufficiently small times. Figure 3 shows vorticity contours at about the last time that the analytical solution is accurate. The dashed curves in this graph represent a simple superposition of single vortex solutions, while the solid curves represent the analytical solution. The difference between the curves represents nontrivial convection effects. It is seen that the redistribution method again very accurately reproduces the analytical curve, despite the presence of very strong convection effects. For more details on this flow we refer to [19]. This clearly shows that the redistribution method correctly computes flows with non-trivial convective processes.

\subsection{Impulsively started cylinder}

A more difficult problem than the previous one is to compute the high-Reynolds number flow over an impulsively started cylinder. This case is however attractive since the literature provides plenty of experimental and numerical data to compare with. To compare with such results, we chose the Reynolds number $(R e)$ based on the diameter and free-stream velocity to be 9500 . In our computation the time step, normalized with cylinder radius and free-stream velocity, was $\Delta t=0.01$; and the maximum distance between neighbors $\sqrt{12 \nu \Delta t}$. The simple treatment of the no-slip boundary conditions at the surface is discussed in [16].

For this computation we start with a single ring of vortices to represent the vortex sheet. As the computation proceeds, our method adds vortices adaptively where there is vorticity. There are about 150000 vortices in the flow at $t=3.00$. Our method does not require remeshing. However, during this computation we did combine vortices if they get very close together. This reduces the number of vortices in the computation, resulting in increased efficiency. Typically, we condensed the vortices once every six time steps; on average this reduces the number of vortices by about $1.5 \%$ each time.

Figure 4a shows the vorticity fields at different times; the non-dimensional times also correspond to the number of radii the cylinder has moved. Figure $4 \mathrm{~b}$ shows the corresponding vorticity fields from a recent high-resolution simulation [9] using particle strength exchange (PSE) scheme [6]. There is an excellent agreement in the vorticity fields, which is non-trivial. 


\subsection{Three-dimensional Stokes flows}

To show that the redistribution method works equally well in three dimensions, we consider two linear problems: diffusion of a pair of opposite vortex poles and Stokes flow due to vortex ring in free space.

Figure 5a shows the diffusion of one of the vorticity components of the vortex pair along the line connecting the vortices. Figure $5 \mathrm{~b}$ shows the isovorticity contours in the right half of the plane through the vortices. The solid lines are exact and the dots are computed; they are in very good agreement. Our computations show that the symmetries in the solution are reproduced very well, even though they are not explicitly enforced.

Figure 6 shows the vorticity fields due to the diffusion of a vortex ring at two different times. It is seen that the ring expands correctly as expected and also preserves the symmetry very well.

A final remark concerns the smoothing function used in the above examples to evaluate the vorticity field. In two-dimensions, we have used Leonard's infinite order smoothing [10] with excellent results. But the equivalent three-dimensional core decays too slowly at large distances to be useful. The reason for the slow decay is the Fourier transform, which is shown as a dotted line in Fig. 7a. It is unity for scaled wave number $k$ less than one and vanishes for larger $k$. This resulting jump singularity causes the slow decay. To obtain exponential convergence, we must smooth the singularity. We choose:

$$
\widehat{\phi}(k)=\frac{1}{\exp ^{2\left(k^{2}-k^{-2}\right)}+1},
$$

where $k$ is the scaled wave number in the radial direction. This produces an infinite order core that converges exponentially at large distance. Since this core cannot readily be evaluated in physical space, we approximated it by a spline interpolant (Fig. 7b).

We are now extending our three-dimensional computations to include convection.

\section{Conclusions}

The vorticity redistribution method is found to be an accurate, robust, truly Lagrangian method. It can handle arbitrary convection without remeshing. It resolves very short scales and has excellent conservative properties. The method extends easily to handle wall boundary conditions, without the need for additional equations.

\section{Acknowledgement}

The authors would like to thank Dr. P. Koumoutsakos of NASA Ames/CTR Stanford University for his permission to reproduce his results in Fig. 4b. This research was supported by AFOSR F49620-93-I-0013, and by NASA NAG-W-2930. Facilities were provided by FMRL and SCRI at FSU.

\section{References}

[1] Almgren, A.S., Buttke, T. and Colella, P., "A Fast Adaptive Vortex Method in Three Dimensions," J. Comp. Phys., 113, pp. 177, 1994. 
[2] Anderson, C.R. and Greengard, C., Vortex Dynamics and Vortex Methods, AMS Lectures in Applied Mathematics, 28, edited by C.R. Anderson and C. Greengard, American Mathematical Society, Rhode Island, 1991.

[3] Anderson, C.R., "An Implementation of the Fast Multipole Method Without Multipoles," SIAM J. Sci. Stat. Comp., 13, pp. 923, 1992.

[4] Caflisch, R.E., Mathematical Aspects of Vortex Dynamics, edited by R.E. Caflisch, SIAM Proceedings Series, 1988.

[5] Carrier, J., Greengard, K. and Rokhlin, V., "A Fast Adaptive Multipole Algorithm for Particle Simulations," SIAM J. Sci. Stat. Comp., 9, pp. 669, 1988.

[6] Degond, P. and Mas-Gallic, S., "The Weighted Particle Method for Convection-Diffusion Equations, Part 1: The Case of an Isotropic Viscosity, Part 2: The Anisotropic Case," Math. Comp., 53, pp. 485, 1989.

[7] Fishelov, D., “A New Vortex Scheme for Viscous Flows," J. Comp. Phys., 86, pp. 211, 1990.

[8] Greengard, L. and Rokhlin, V., "On the Efficient Implementation of the Fast Multipole Algorithm," Yale University, Dept. of Computer Science Report No. YALEU/DCS/RR-602, 1988 (unpublished).

[9] Koumoutsakos, P. and Leonard, A., "High-Resolution Simulations of the Flow Around an Impulsively Started Cylinder Using Vortex Methods," J. Fluid Mech., 296, pp. 1, 1995.

[10] Leonard, A., "Vortex Methods for Flow Simulation," J. Comp. Phys., 37, pp. 289, 1980.

[11] Leonard, A., "Computing Three Dimensional Incompressible Flows With Vortex Elements," Ann. Rev. Fluid Mech., 17, pp. 523, 1985.

[12] Puckett, E.G., "Vortex Method: An Introduction and Survey of Selected Research Topics," Incompressible computational fluid dynamics, edited by M.D. Gunzburger and R.A. Nicolaides, Cambridge Univ. Press, pp. 335, 1993.

[13] Sarpkaya, T., "Vortex Element Methods for Flow Simulations," Adv. Appl. Mech., 31, pp. $113,1994$.

[14] Shankar, S. and van Dommelen, L., "A New Diffusion Procedure For Vortex Methods," J. Comp. Phys. 126, 1996, to appear.

[15] Shankar, S., Wang, S.-C., and van Dommelen, L.L., "Simulating Diffusion in Vortex Methods Using a Vorticity Redistribution Technique," Forum on Vortex Methods for Engineering Applications, Sandia National Laboratory, Albuquerque, New Mexico, pp. 105, 1995.

[16] Shankar, S., A Mesh-Free Vortex Method, Ph.D thesis, Florida State University, 1996 (in preparation).

[17] van Dommelen, L.L. and Rundensteiner, E.A., "Fast, Adaptive Summation of Point Forces in the Two-Dimensional Poisson Equation," J. Comp. Phys., 83, pp. 126, 1989.

[18] van Dommelen, L.L., "A Vortex Redistribution Technique," FMRL Report TR-3, Department of Mechanical Engineering, Florida State University, 1989 (unpublished). 
[19] van Dommelen, L. and Shankar, S., "Two Counter-Rotating Diffusing Vortices," Phys. Fluids A, 7, pp. 808, 1995.

[20] van Dommelen, L. and Shankar, S., "The Separation Singularity at Infinite and Finite Reynolds Numbers," (in preparation).

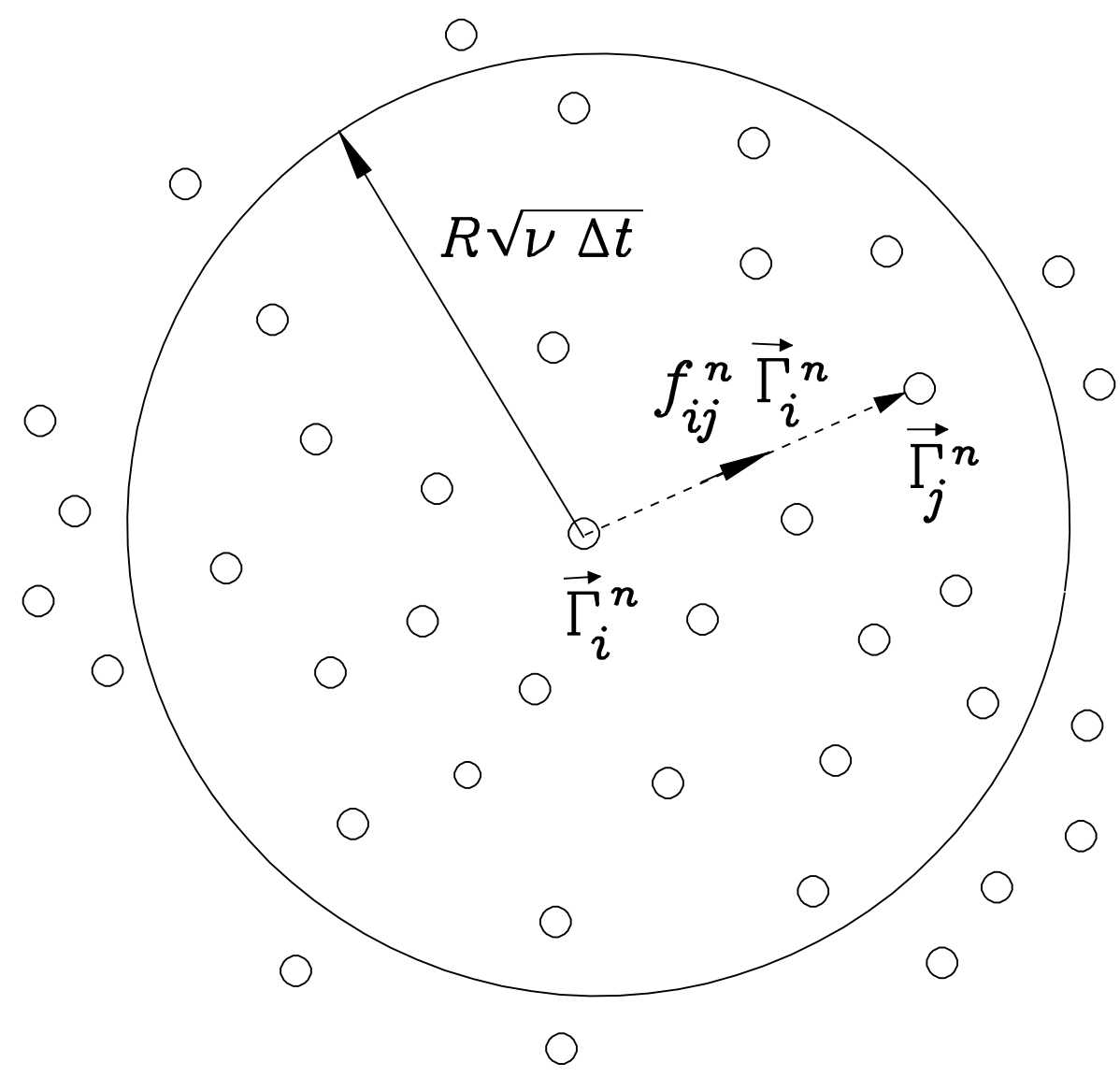

Figure 1: Redistribution of the circulation of a vortex $\Gamma_{i}$ of a vortex $i$. 

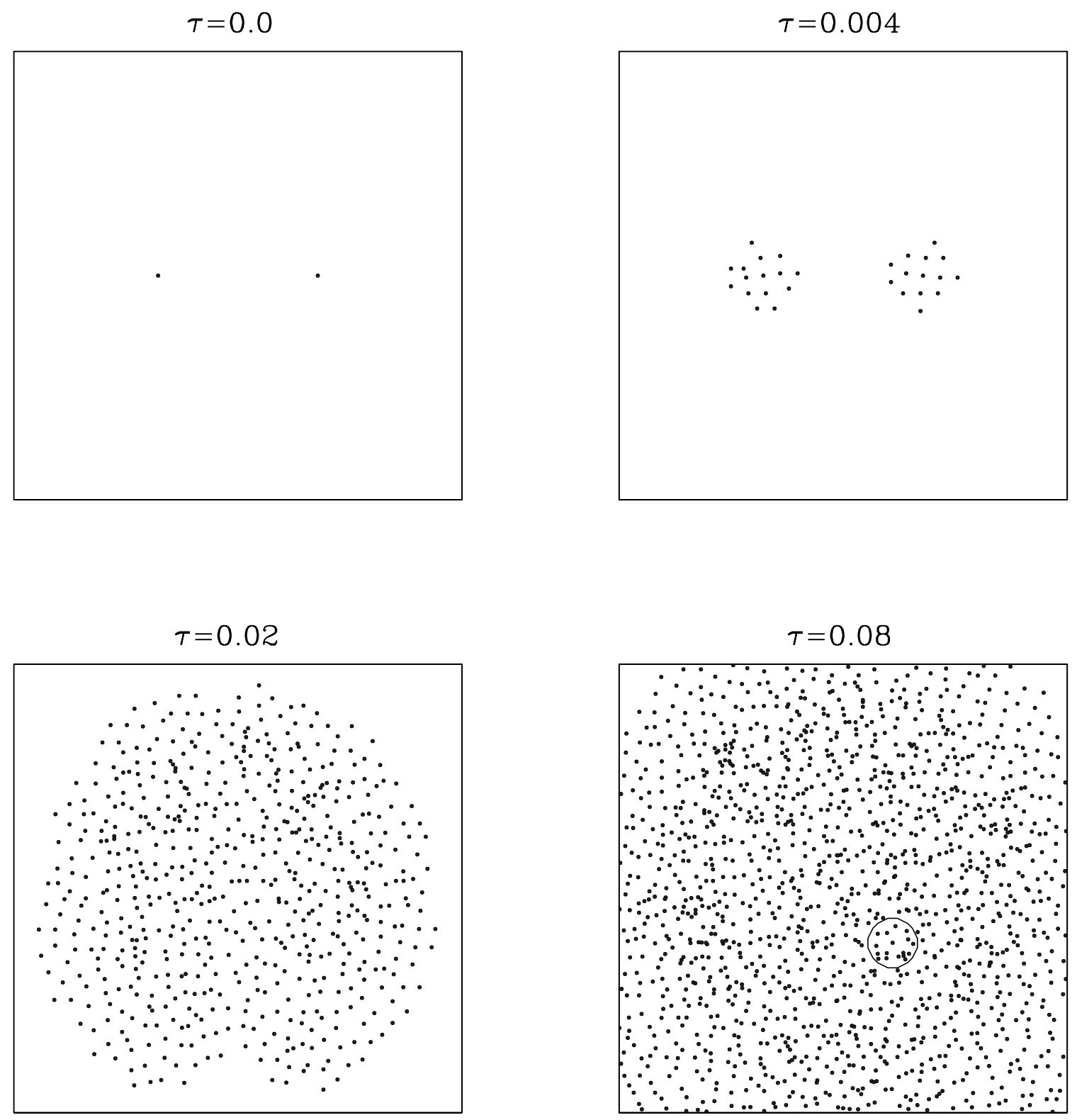

Figure 2: Computational vortices to compute the diffusion of counter-rotating vortex pair at $R e=$ 50. 


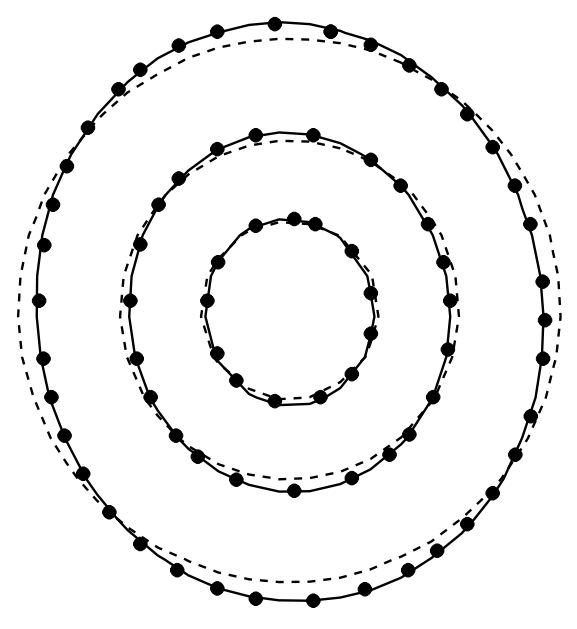

Figure 3: Isovorticity contours $\omega=40,24,8$, for a counter-rotating vortex pair for $R e=50$ at time $\tau=0.01025$. The dashed and solid lines represent orders of approximation in the analytic expansion. Dots are computed values. 

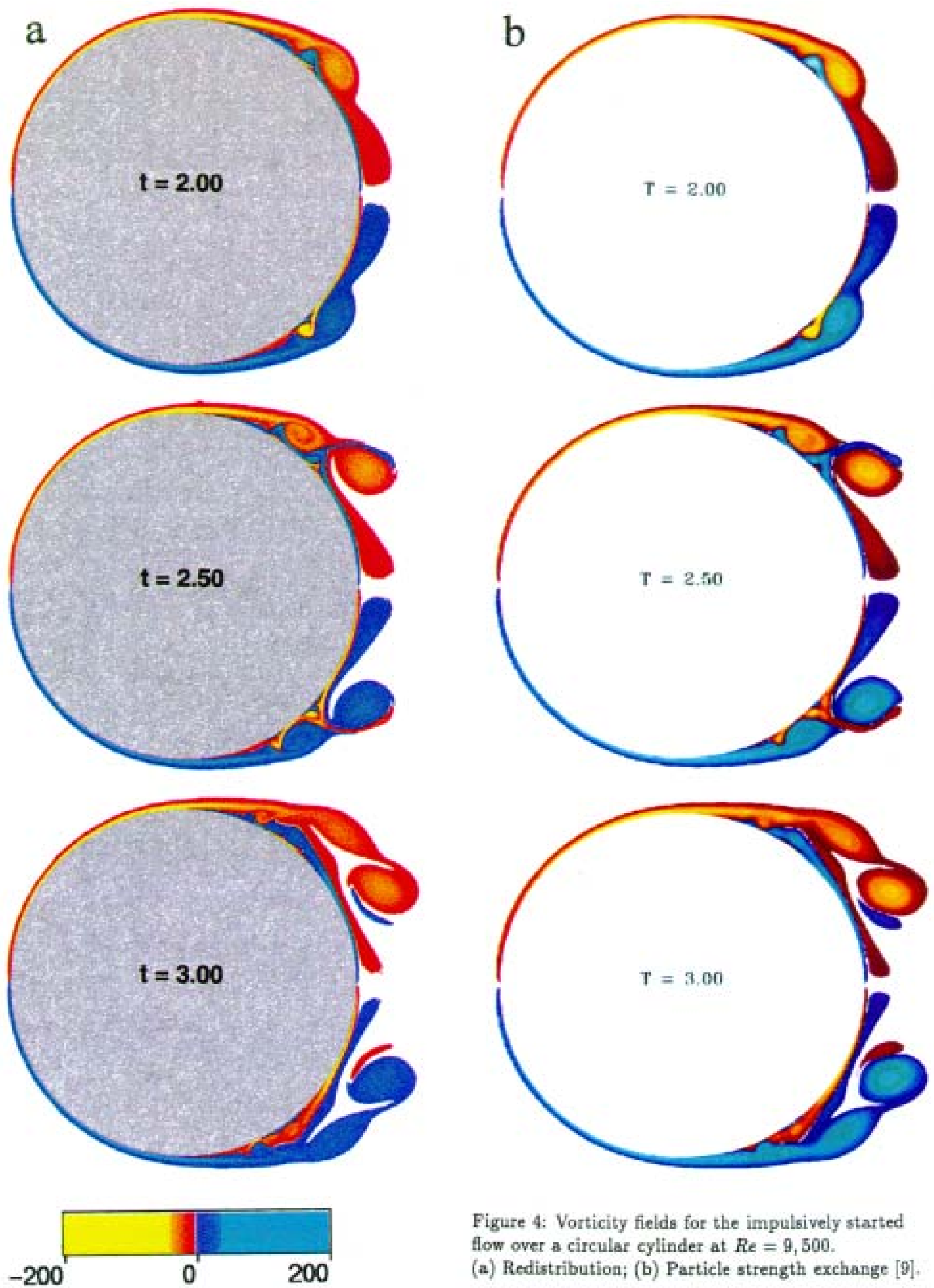

Figure 4: Vorticity fields for the impulsively started flow over a circular cylinder at $R e=9,500$.

(a) Redistribution; (b) Particle strength exchange [9]. 

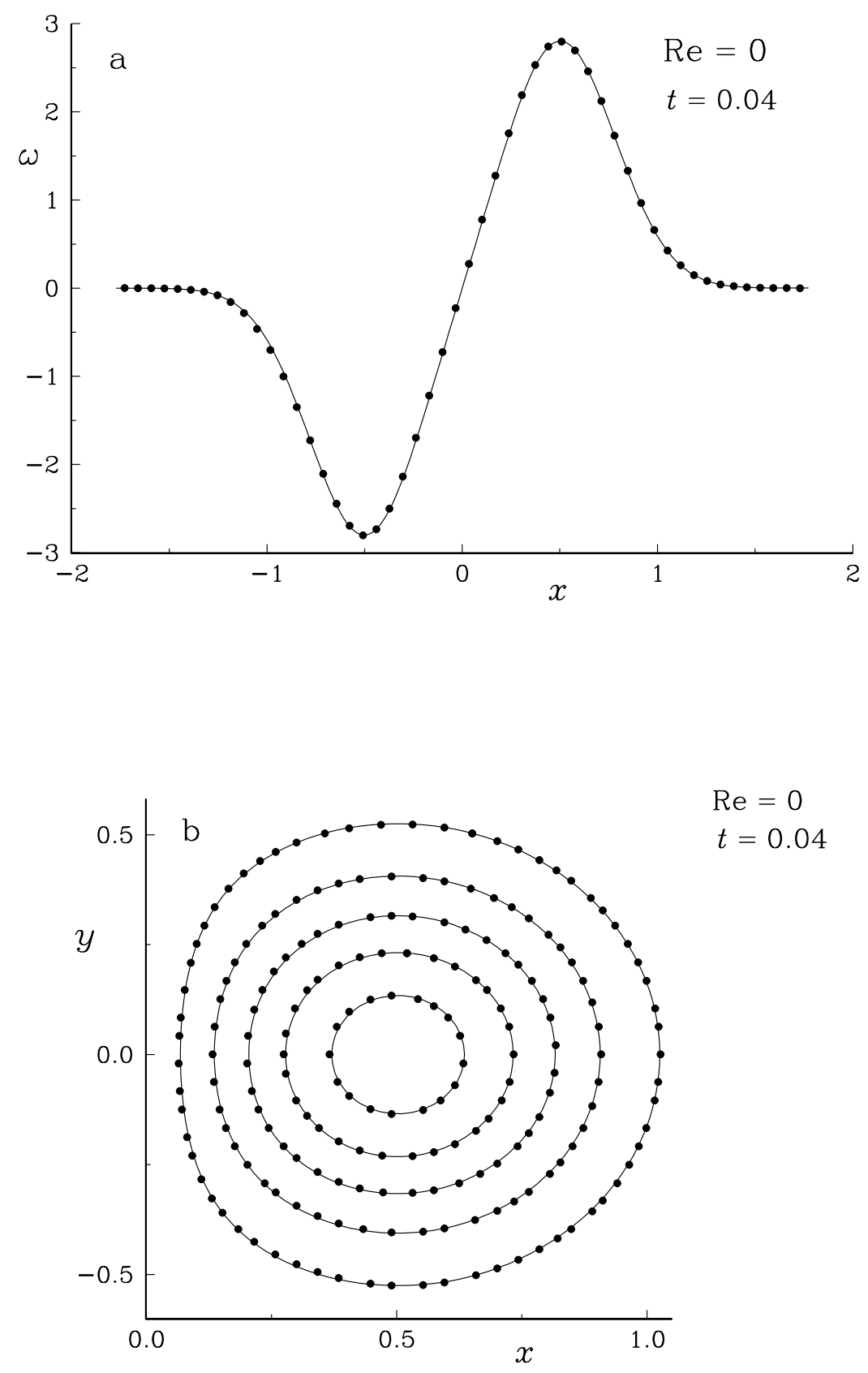

Figure 5: Vorticity for three-dimensional diffusion of a pair of vortex poles: (a) Along a line through the vortices; (b) Isovorticity contours $\omega=0.5,1.0,1.5,2.0,2.5$, in the plane of the vortices. The solid lines are exact and dots are computed values. 

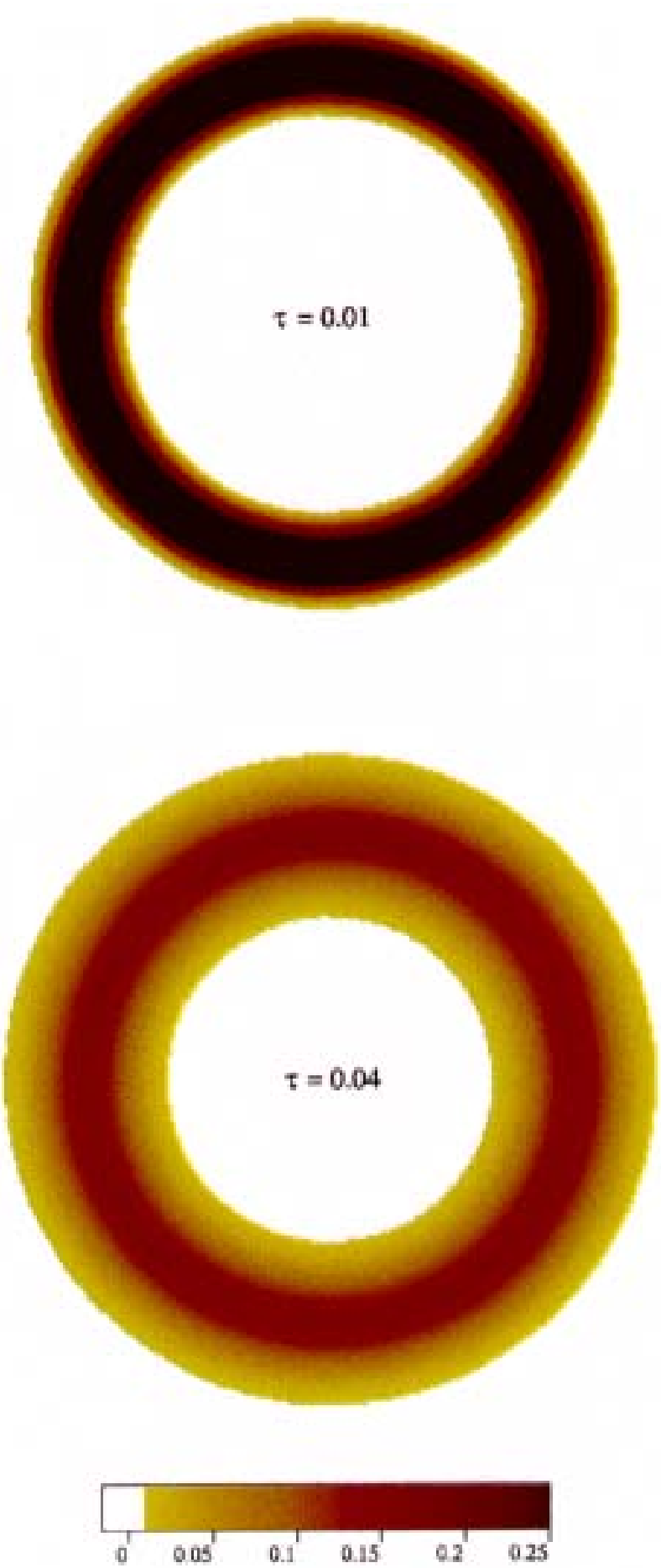

Figure 6: Vorticity field of a diffusing vortex ring at $R e=0$. 

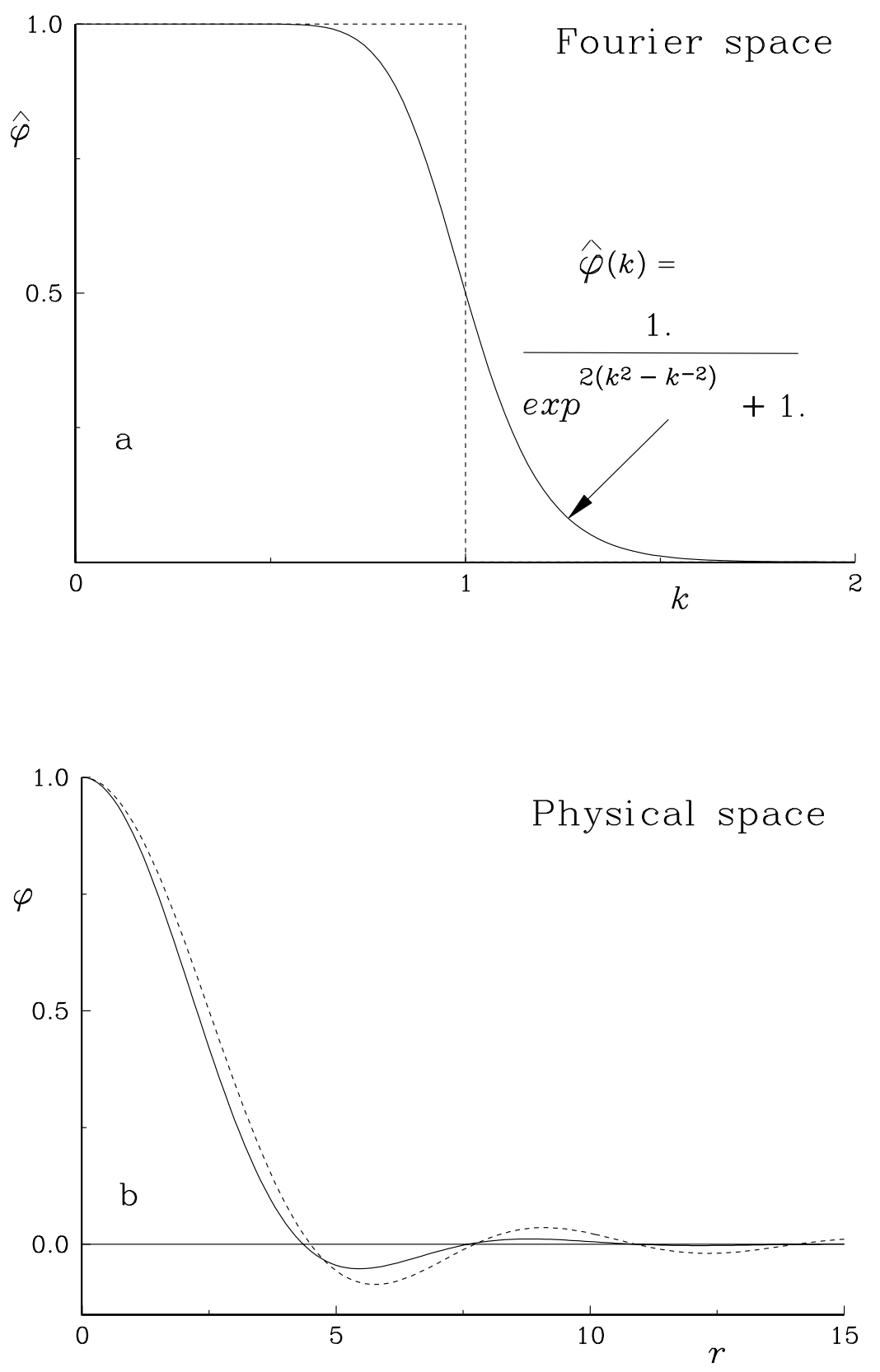

Figure 7: Smoothing functions in (a) Fourier space; (b) Physical space. Broken lines are nonconvergent smoothing; solid lines are modified smoothing. 